\title{
Transport properties of electron swarms in tetrahydrofuran under the influence of an applied electric field
}

\author{
N. A. Garland,,${ }^{1, *}$ M. J. Brunger, ${ }^{2,3}$ G. Garcia, ${ }^{4}$ J. de Urquijo, ${ }^{5}$ and R. D. White ${ }^{1}$ \\ ${ }^{1}$ ARC Centre for Antimatter-Matter Studies, School of Engineering and Physical Sciences, James Cook University, Townsville 4810, Australia \\ ${ }^{2}$ ARC Centre for Antimatter-Matter Studies, School of Chemical and Physical Sciences, Flinders University, GPO Box 2100 , \\ Adelaide, SA 5001, Australia \\ ${ }^{3}$ Institute of Mathematical Sciences, University of Malaya, 5063 Kuala Lumpur, Malaysia \\ ${ }^{4}$ Instituto de Física Fundamental, Consejo Superior de Investigaciones Científicas, Madrid 28006, Spain \\ ${ }^{5}$ Instituto de Ciencias Físicas, Universidad Nacional Autónoma de México, 62251 Cuernavaca, Morelos, Mexico
}

(Received 24 September 2013; published 26 December 2013)

\begin{abstract}
Using an almost complete set of electron impact cross sections for scattering from the important biomolecule tetrahydrofuran (THF), compiled as a part of this study, swarm transport coefficients are determined by solving the Boltzmann's equation over the range of applied reduced fields from 0.01 to $10000 \mathrm{Td}$. The present investigation highlights the experimental issues associated with, and the real need for, measurements of the corresponding THF transport coefficients, so that the self-consistency of our proposed cross section set might be evaluated.
\end{abstract}

DOI: 10.1103/PhysRevA.88.062712

PACS number(s): 34.80.-i

\section{INTRODUCTION}

Ionizing radiation, used in many imaging and therapeutic technologies, liberates large numbers of secondary electrons $\left(\sim 10^{4}\right.$ electrons per $\mathrm{MeV}$ deposited) along its path, with secondary electron energy distributions being typically less than 20-30 eV. These low-energy electrons thermalize in human tissue through a variety of energy deposition processes in biomolecules such as water, the sugars, and the DNA bases. Although low in energy, subionization electrons have been shown to be a source of DNA damage [1]. The process of dissociative electron attachment (DEA) can lead to single and double strand breaks directly, or indirectly through the formation of free radicals which interact with the DNA. The pioneering study of Sanche and co-workers [1,2] has subsequently motivated much research into electron collision processes with subunits and constituents of the moieties that constitute DNA and RNA.

A quantitative understanding of the transport of low-energy secondary electrons in human tissue is key to understanding radiation damage and informing dosimetry models. Consequently, requisite databases are required for electron-induced processes in biomolecules for track simulations and transport studies. Water is generally assumed as the surrogate for human tissue, and recently full sets of cross sections have been developed and tested for electron-water interactions in the gas phase [3,4]. Recent work has suggested that such gas-phase studies can be adapted to the soft-condensed phase through appropriate modifications using pair correlations functions $[5,6]$. While there has been much progress in the study of electron-induced processes in the sugars and DNA bases, they have not developed to the same level of completeness as is the case for water. While DNA is currently not convenient to study, tetrahydrofuran $\left(\mathrm{THF}, \mathrm{C}_{4} \mathrm{H}_{8} \mathrm{O}\right)$ has been investigated as a model for low-energy electron interactions with 2deoxyribose, a sugar that links phosphate groups in the DNA backbone. Considerable progress has been made in recent

\footnotetext{
*nathan.garland@my.jcu.edu.au
}

times establishing individual cross sections for electron impact processes in THF, at both the integral and differential levels. Experimentally, there have been measurements of the total [7,8], (quasi-) elastic [9-14], vibrational [10,15,16], electronic excitation [17,18], and ionization $[19,20]$ cross sections over a range of energies. Likewise, theoretical treatments using $R$-matrix methods [21], the Schwinger variational method $[13,22]$, the complex Kohn variational method [23] and binary encounter-Bethe approach [24], the independent atom model (IAM) [24], and the recent IAM-screening corrected additivity rule (SCAR) treatment $[25,26]$ have provided complementary information to the experimental data. The levels of agreement vary between the various techniques and theories for many of the processes, and this paper aims to propose cross sections for THF utilizing the strengths of the various approaches where possible.

Quantitative modeling of electron transport in biological matter requires the compilation of the best available set of cross sections for all collisional processes (e.g., elastic, rotations, vibrations, and so on). While this is generally based on a critical assessment of available experimental studies and theoretical calculations, it also requires interpolation and extrapolation of those cross sections to the energies required for the simulation. For any proposed cross-section set, a key question remains, however: Is the resulting set of cross sections complete and accurate? Experimental swarm physics $[27,28]$ continues to play an important role in this regard $[4,29]$. In swarm experiments, electrons are released into a drift chamber containing the target gas with a prescribed pressure and temperature. These electrons are acted upon by an applied uniform electric field $E$, which drives them out of thermal equilibrium with the background gas into a nonequilibrium steady state where the power input from the field is balanced by the power deposited in collisions. Currents are sampled and interpreted in terms of transport coefficients (e.g., mobility and diffusion coefficients) as a function of the applied reduced field $E / n_{0}$ where $n_{0}$ is the target number density. The macroscopic transport coefficients can be interpreted in terms of the microscopic electron impact cross sections, and hence by varying $E / n_{0}$ we can effectively 
sample the energy dependence of the cross sections. Swarm experiments vary from crossed-beam experiments in that (i) they are multiple scattering experiments as opposed to the single scattering beam experiments, requiring particle, momentum, and energy balance for the entire ensemble of electrons in the swarms; and (ii) the velocity distribution of the electrons is unknown and must be determined from transport theory in order to link the cross sections to the transport coefficients.

In Sec. II we survey existing total, integral, and differential cross sections in THF and propose an almost complete set of electron impact cross sections for THF, including elastic, rotational, vibrational, electronic, ionization, DEA, and neutral dissociation processes in the energy range $\sim 0-300 \mathrm{eV}$. In Sec. III we discuss issues we have found in attempts to measure swarm transport coefficients in THF and present calculated transport coefficients using a Boltzmann equation treatment aimed to motivate further experimental swarm studies in THF. Finally, in Sec. IV some conclusions from the present investigation are drawn.

\section{CROSS SECTIONS IN THF}

In this section we develop a recommended set of cross sections for electrons in THF. We restrict ourselves to the energy region less than $300 \mathrm{eV}$. Above this energy, agreement between theory and experiment is sufficiently good that theory can, in general, be used $[25,26]$. We focus on the development of a set of cross sections for implementation into a Boltzmann equation transport theory (or Monte Carlo simulations) and hence restrict ourselves to discussions of the integral cross sections (ICSs), while the differential cross sections are sufficiently represented through implementation of the integrated forms including the momentum transfer cross sections (MTCSs).

\section{A. Grand total cross-section set}

The grand total cross section (GTCS) for THF consists of contributions from the elastic $\sigma_{\text {elas }}$, rotational $\sigma_{\text {rot }}$, vibrational $\sigma_{\text {vib }}$, DEA $\sigma_{\text {DEA }}$, electronic $\sigma_{\text {elec }}$, ionization $\sigma_{\text {ion }}$, and neutral dissociation $\sigma_{\text {neutral }}$ ICSs. The GTCS has been extensively studied both experimentally $[7,8]$ and computationally $[25,26]$. We propose a GTCS based on the most recent set of Chiari et al. [26], although we have modified the Chiari et al. data to account for the resonance at approximately $6 \mathrm{eV}$ that has been observed in the previous experimental measurements $[7,8]$. For sub-1-eV energies, the proposed GTCS is the sum of the proposed elastic ICS from Sec. IIB and the rotational ICS from Sec. IIF, which are from the same calculations of Chiari et al. [26] and from our own application of the first Born approximation. To construct the resonance in the proposed set, the slopes on either side of the measured resonances in the GTCS from references $[7,8]$ were computed and found to be similar. This characteristic of the resonance from both data sets was subsequently used to splice in the known resonance into the Chiari et al. data set. The theoretical data of Chiari et al. was chosen in lieu of experimental data because of the sufficient magnitude their GTCS has, such that when all ICSs are subtracted from the GTCS the result

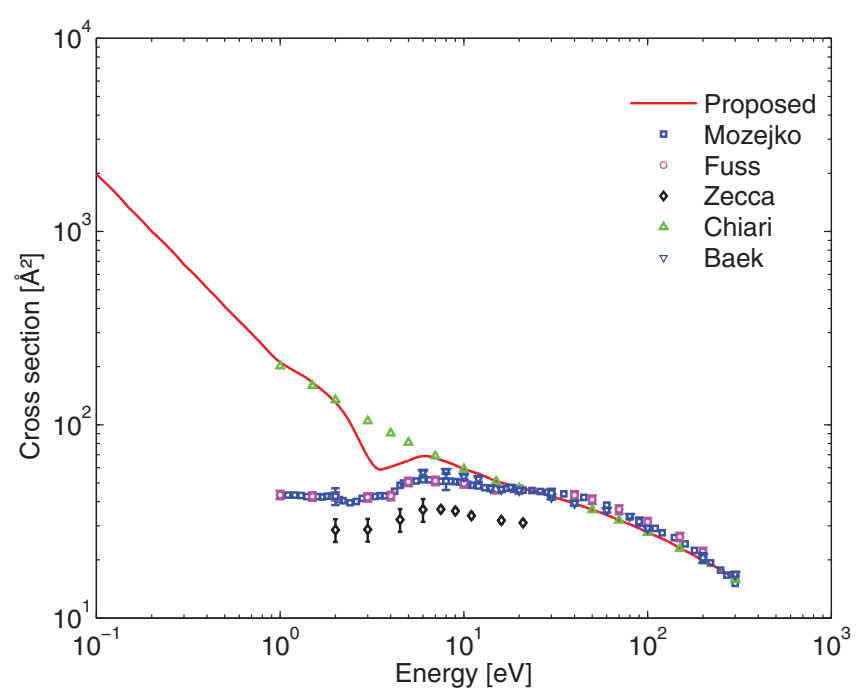

FIG. 1. (Color online) Proposed GTCS for electron scattering in THF, as compared with existing GTCS data (Mozejko et al. [8]; Fuss et al. [25]; Zecca et al. [7]; Chiari et al. [26]).

is non-negative and therefore physical. Using experimental GTCS data yielded negative cross sections for the proposed neutral dissociation cross section and was therefore found unfit for the proposed GTCS. This observation is almost certainly due to the angular discrimination effect on the measured GTCS, causing them to be a lower bound on their true values [30,31]. The resulting proposed GTCS for electrons in THF is presented in Fig. 1, where they are compared with other experimental and theoretical data.

\section{B. Elastic cross-section set}

There have been a number of measurements of the elastic differential and ICSs for a variety of energy and angular ranges [9-14]. These have been complemented by theoretical calculations using the $R$ matrix [21], Schwinger variational method [22] (more recently accounting for the long-range scattering by the strong permanent electric dipole of THF [13]), and the independent atom model (IAM-SCAR) [19,25,26]. Multiple elastic ICSs were sourced from Fuss et al. [19], Colyer et al. [9], Baek et al. [12], Dampc et al. [14], and Gauf et al. [13] in order to construct the proposed elastic ICS by capturing the relative strengths of the various approaches. For energies higher than $50 \mathrm{eV}$, there is relatively good agreement between results from the various techniques and so a combination of Baek et al. [12] and the theoretical elastic ICS from Fuss et al. [19] was used. Similarly, but now for energies between $10-50 \mathrm{eV}$, the available measured data [9,12-14] are all in good accord, and so the Colyer et al. results were employed to construct our proposed set here. A recent erratum by the CSU-Fullerton group [32] indicated that their water cross sections were, on average, $15 \%$ larger than they should be with the effect being greater at the lower energies they studied. As the THF results of Gauf et al. [13] were made with the same apparatus, and using the same procedures and techniques as their $\mathrm{H}_{2} \mathrm{O}$ work, the elastic ICSs of Gauf et al. were scaled down in the $1-10-\mathrm{eV}$ region by the ratio of the 


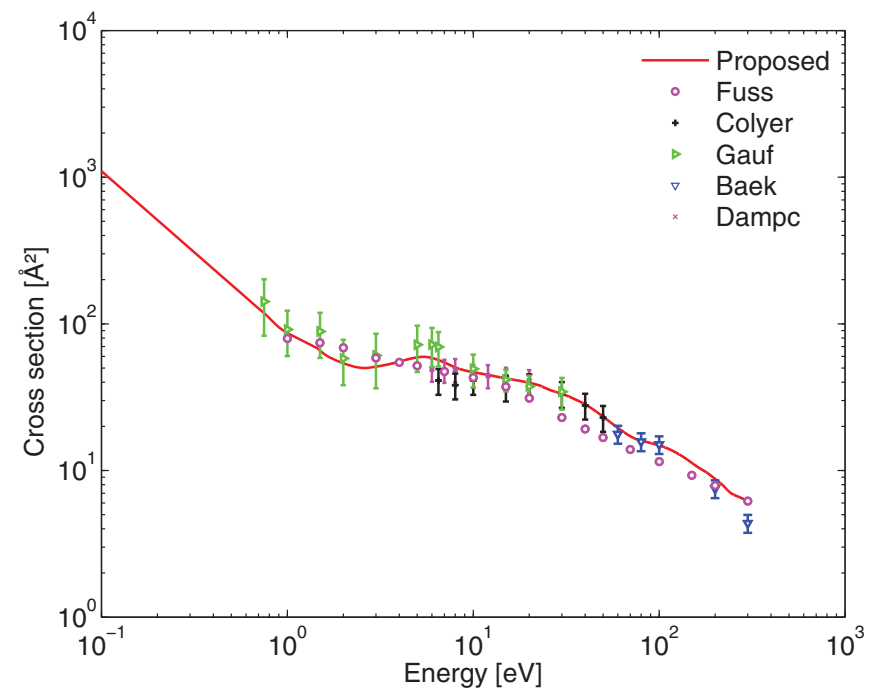

FIG. 2. (Color online) Proposed elastic ICS for electron scattering in THF, compared with existing data from which the set was constructed (Colyer et al. [9]; Baek et al. [12]; Dampc et al. [14]; Gauf et al. [13]; Fuss et al. [19]).

Colyer et al. ICS to the Gauf et al. ICS at $10 \mathrm{eV}$. The proposed set between 1 and $10 \mathrm{eV}$ was then constructed from that scaled data of Gauf et al. Finally, for energies between 0.1 and $1 \mathrm{eV}$, we have used the energy dependence of the $\mathrm{H}_{2} \mathrm{O} R$-matrix results from Tennyson et al. [33] to assist us to construct our proposed set. As dipole scattering is expected to dominate at those lower energies, and as the dipole moments of THF and $\mathrm{H}_{2} \mathrm{O}$ are not dissimilar, we believe this approach should be quite physical. In summary, the proposed elastic ICS is compared with the data used to construct it $[9,12-14,19]$ in Fig. 2. An equivalent process was also employed to establish the elastic MTCS displayed in Fig. 3.

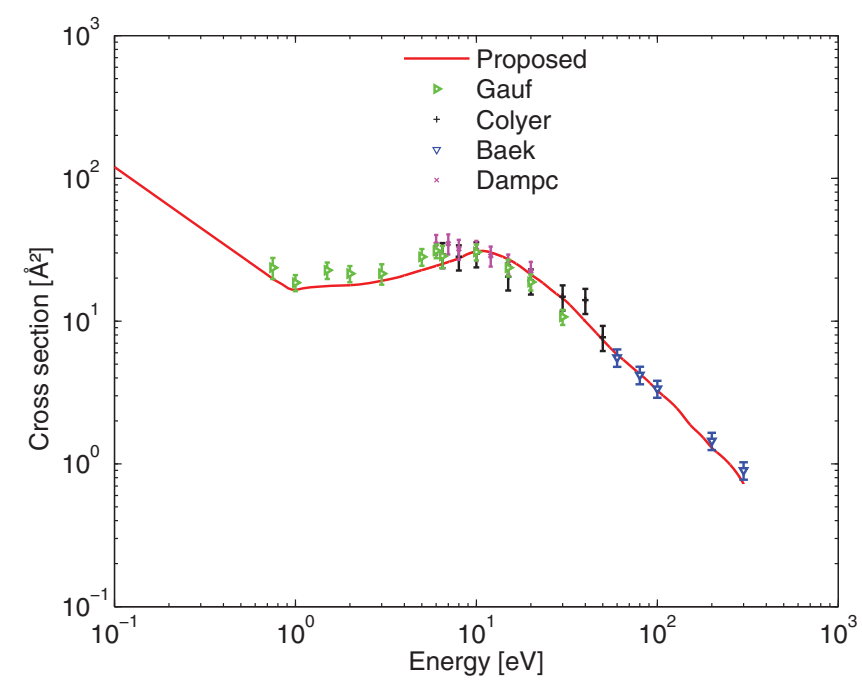

FIG. 3. (Color online) Proposed elastic MTCS for electron scattering in THF, compared with existing data from which the set was constructed (Baek et al. [12]; Dampc et al. [14]; Gauf [13]; Colyer [9]).

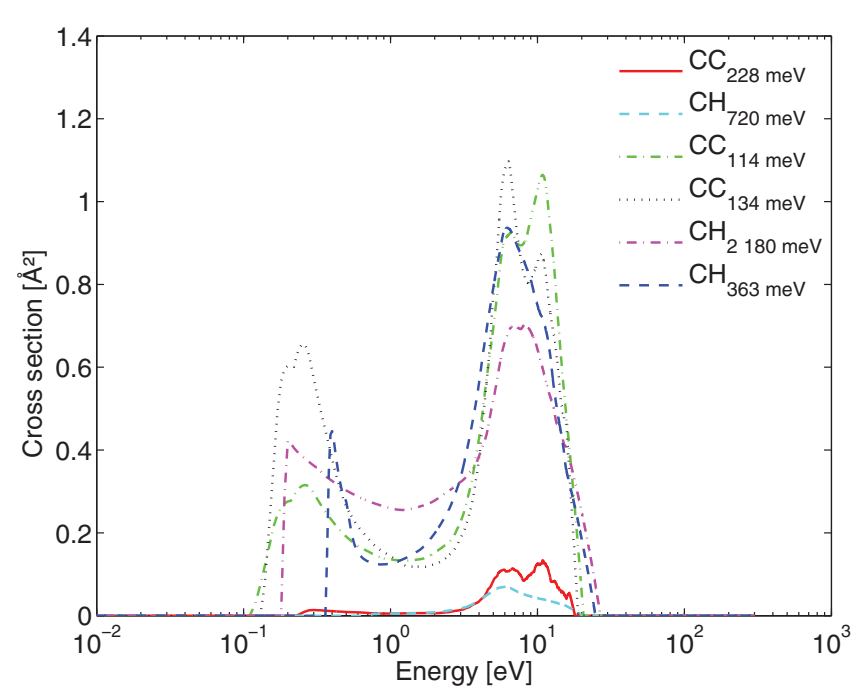

FIG. 4. (Color online) Proposed vibrational ICS for electron scattering in THF. See legend for further details.

\section{Vibrational excitation cross-section set}

Electron impact vibrational excitation cross sections for scattering in THF have been measured by various groups $[10,15,16]$. In this work, we propose a set of six ICSs for the identified vibrational modes of THF in the work from Allan [10]. In general, if the angular distribution of a DCS is isotropic at some incident electron energy $\varepsilon$, then for any scattering angle $\theta=\theta_{0}$ the ICS is simply given by $\operatorname{ICS}(\varepsilon)=$ $4 \pi \operatorname{DCS}\left(\varepsilon, \theta_{0}\right)$. The DCS of Khakoo et al. [16] suggest that, with the exception of their lowest energy work at $2 \mathrm{eV}$, the assumption of isotropic angular distributions for the DCS is quite adequate. Nonetheless, in an attempt to lessen the effects of any anisotropic scattering, we have averaged the measured vibrational excitation functions of Allan [10] for each respective mode, at $\theta=45^{\circ}, 90^{\circ}, 135^{\circ}$, and $180^{\circ}$, before multiplying by $4 \pi$ to generate an $\operatorname{ICS}(\varepsilon)$ in each case.

The results from our analysis are displayed in Fig. 4. For comparison, in Fig. 5 we plot the vibrational cross section of Khakoo et al. [16], whose measurements were conducted at a lower energy resolution and therefore incorporate several of the individual modes measured by Allan [10] with our relevant summed modes proposed in Fig. 4. We see in Fig. 5, to within the stated uncertainties from our analysis and the measurements of Khakoo et al., that the level of agreement between them is generally quite good, with our ICS being systematically smaller in magnitude. Nonetheless, the results embodied in Fig. 5 give us some confidence in the validity of our approach in determining the THF vibrational ICSs.

For implementation into transport theories, and or Monte Carlo simulations, it is important to have differentiated (rather than lumped) processes, where possible, to ensure that the relevant threshold energies are included in the transport results [4]. This is the main reason we prefer using the data of Allan [10] to that of Khakoo et al. [16]. 


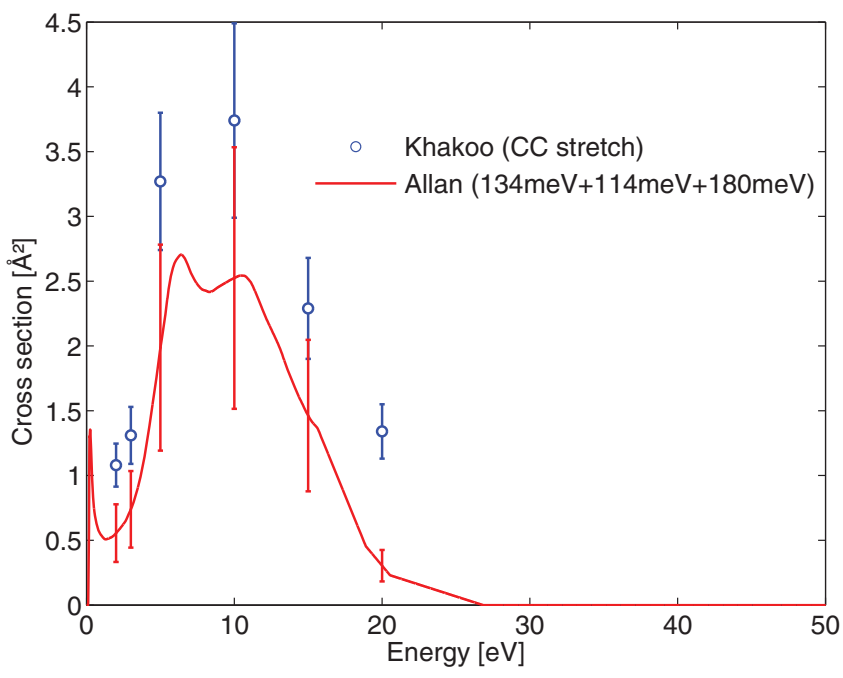

FIG. 5. (Color online) Comparison of a subset of our recommended ICS for electron-induced vibrational processes in THF [10], with the summed modes of Khakoo et al. [16].

\section{Nonconservative cross-section sets: Dissociatative electron attachment and ionization}

There have been limited investigations of electron impact ionization in THF. Here we note the theoretical studies from Mozejko and Sanche [24], and the experimental studies of Dampc et al. [20] and Fuss et al. [19]. In this investigation we implement the calculated ionization ICS of Mozejko and Sanche, with an ICS of $0 \AA^{2}$ at the $9.55-\mathrm{eV}$ ionization threshold for THF from Dampc et al. As the data of Fuss et al. only extends down to $50 \mathrm{eV}$, and the calculations of Mozejko and Sanche are in good agreement with the measurements of Fuss et al., where they overlap in energy, we prefer to use the data of Mozjeko and Sanche for the full energy range. Data from Dampc et al. [20] shows good agreement with other reported ionization ICS data above approximately $70 \mathrm{eV}$ but, below this energy, variation in the Dampc et al. results from the other reported data led to the Mozejko and Sanche ICS being implemented in the proposed ICS. Our proposed ionization ICS is compared with the available theoretical and experimental results in Fig. 6.

While the DEA cross section for THF is very small in magnitude, we nonetheless include it in our analysis by adopting that reported in Aflatooni et al. [34] as our proposed set. That data are plotted in Fig. 7.

\section{E. Electronic-state excitation cross-section sets}

Investigations of electronic-state excitation of THF are restricted to the experimental studies of Do et al. [17] and Zubek et al. [18]. The first three Rydberg bands of ICSs for electronic excitation $\left(\sigma_{\text {elec }}^{(1)}, \sigma_{\text {elec }}^{(2)}, \sigma_{\text {elec }}^{(3)}\right)$ have been reported by Do et al. [17]. Cross sections for the three higher level bands of electronic excitations, which are apparent in the energy-loss spectra in Do et al. and converge to the ionization threshold of THF, are not available, although the threshold energies for the processes are known [17]. The remaining

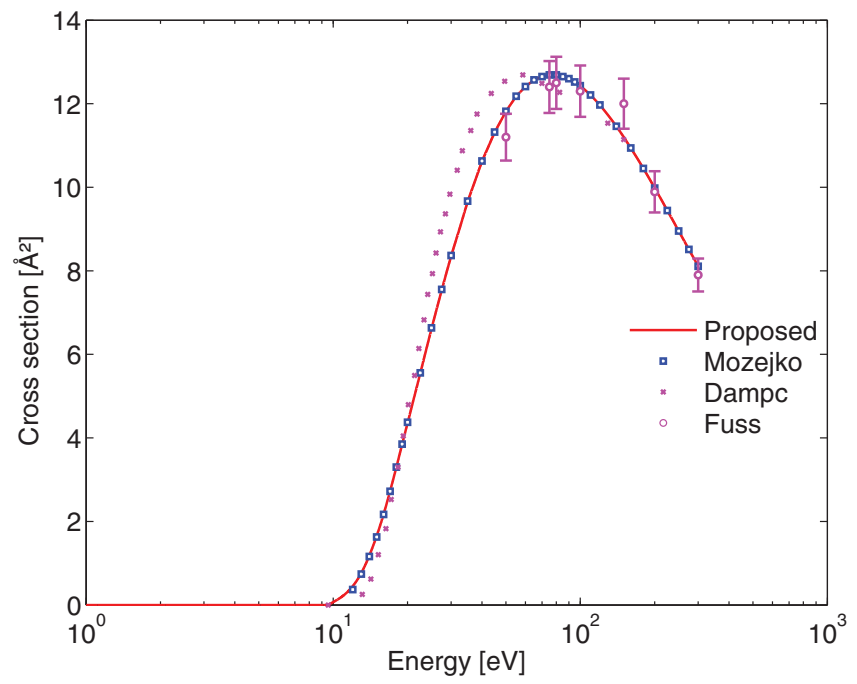

FIG. 6. (Color online) Comparison of the proposed ionization cross sections for electron scattering in THF with the available theoretical calculations of Mozejko and Sanche [24] and Dampc et al. [20] and the measured data from Fuss et al. [19].

three electronic mode ICS $\left(\sigma_{\text {elec }}^{(4)}, \sigma_{\text {elec }}^{(5)}, \sigma_{\text {elec }}^{(6)}\right.$ ) were assumed to have the same functional form as $\sigma_{\text {elec }}^{(3)}$. Their magnitudes were estimated by adding, respectively, 10\%, 20\%, and $30 \%$ of the maximum value of $\sigma_{\text {elec }}^{(3)}$ to the cross section $\sigma_{\text {elec }}^{(3)}$. It should be noted that these scaling factors are not random; they were estimated on the basis of the energy-loss spectra in Do et al. [17] and the many other energy-loss data measured by the Flinders group. While they do represent, in this use, a form of average scaling factor, we believe them to be accurate to within the typical uncertainties of experimental electronic-state ICS determination $(45 \%-50 \%)$.

These cross sections $\left(\sigma_{\text {elec }}^{(4)}, \sigma_{\text {elec }}^{(5)}, \sigma_{\text {elec }}^{(6)}\right)$ were also shifted to their relevant thresholds for each of the electronic excitation

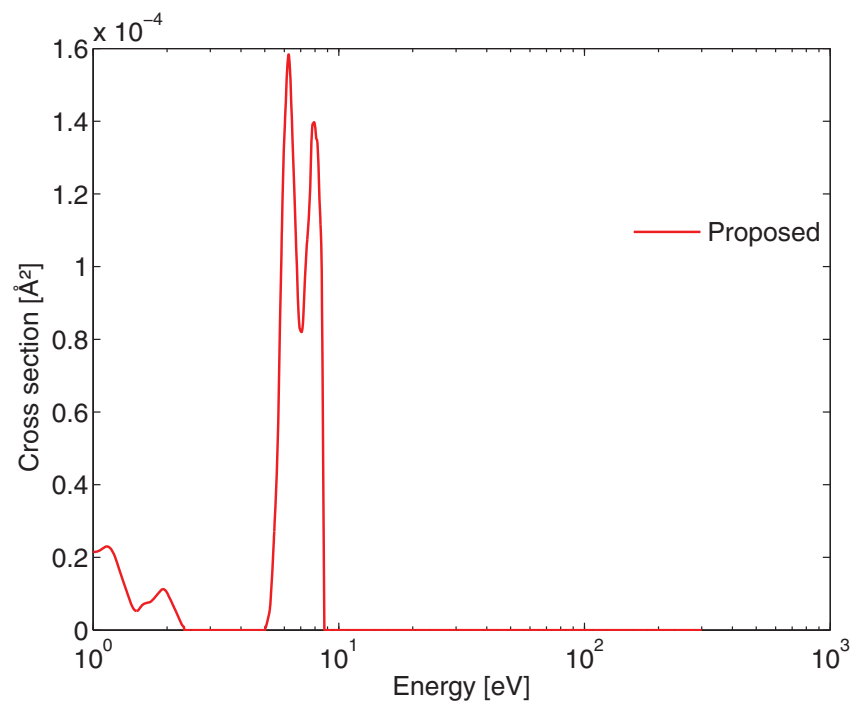

FIG. 7. (Color online) Dissociative electron attachment crosssection for THF, as adopted from [34]. 


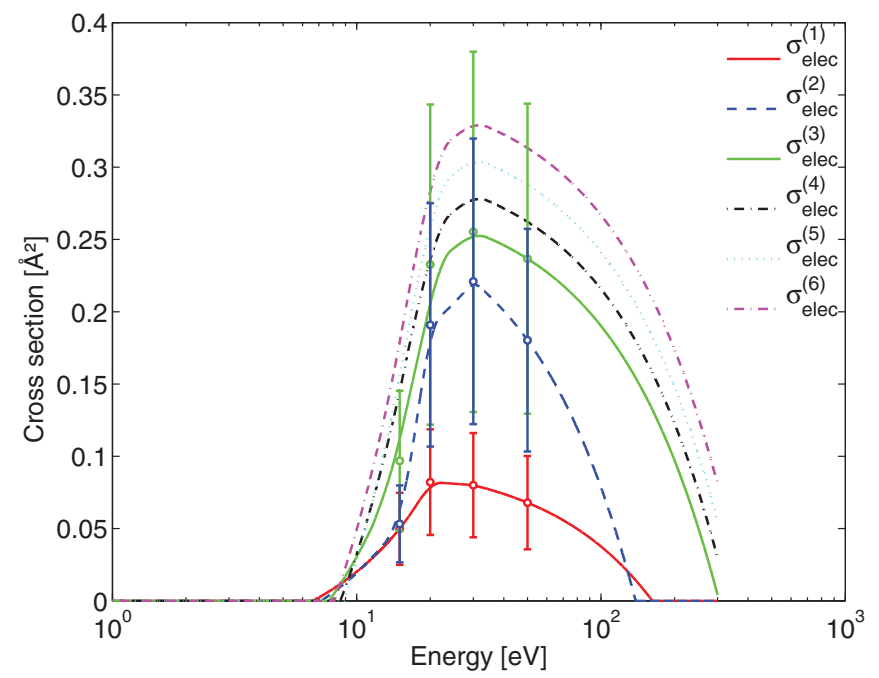

FIG. 8. (Color online) Proposed electronic excitation ICS for electron scattering from THF, as determined using the data of Do et al. [17]. The excitation energies of each electronic state are as follows: state $1=6.6 \mathrm{eV}$; state $2=7.15 \mathrm{eV}$; state $3=7.7 \mathrm{eV}$; state $4=$ $8.57 \mathrm{eV}$; state $5=8.89 \mathrm{eV}$; state $6=8.1 \mathrm{eV}[17]$.

processes. The proposed set of electronic-state excitation ICSs are displayed in Fig. 8.

\section{F. Rotational excitation cross-section set}

Using the procedure suggested by Jain [35], rotational excitation ICS for a free electric dipole are calculated in the framework of the first Born approximation (FBA) for energies in the range $0.1-300 \mathrm{eV}$. Calculations indicate that the lowest threshold for rotational excitation in THF is less than $1 \mathrm{meV}$ and with an average excitation threshold of $1.205 \mathrm{meV}$. Note that the rotational excitation energies $\left(\Delta E_{\mathrm{rot}}=E_{J}-E_{J^{\prime}}\right)$ are calculated from the rotational constants derived from the moments of inertia $\left(I_{x}, I_{y}, I_{z}\right)$ calculated for the same geometrical configuration of the molecule (atomic coordinates taken from the US National Library of Medicine [36]) that we used for the SCAR procedure. Rotational states $\left(E_{J}\right)$ are then approximated by $E_{J}=\langle B\rangle J(J+1)$, where $\langle B\rangle$ is the mean value of the rotational constants $B_{x}, B_{y}$, and $B_{z}$, with $B_{i}=\frac{\hbar^{2}}{2 I_{i}}$. In our proposed set, we have characterized the rotational process by a single (lumped) ICS with a representative threshold energy of $1.205 \mathrm{meV}$. The present ICS is compared with corresponding results extracted by Fuss et al. [25] and with a similar Born-dipole calculation from Chiari et al. [26]. Where an energy overlap is found, the present rotational cross sections are in good agreement with those of Chiari et al., as seen in Fig. 9.

\section{G. Unknown cross section: Neutral dissociation}

To estimate the unknown remnant ICS for electron collisions with THF, the known and proposed cross sections (elastic, rotational, DEA, vibrational, electronic excitation, and ionization) were subtracted from the GTCS set proposed in Fig. 1. The remaining cross section, after this subtraction process, should correspond to that for neutral dissociation.

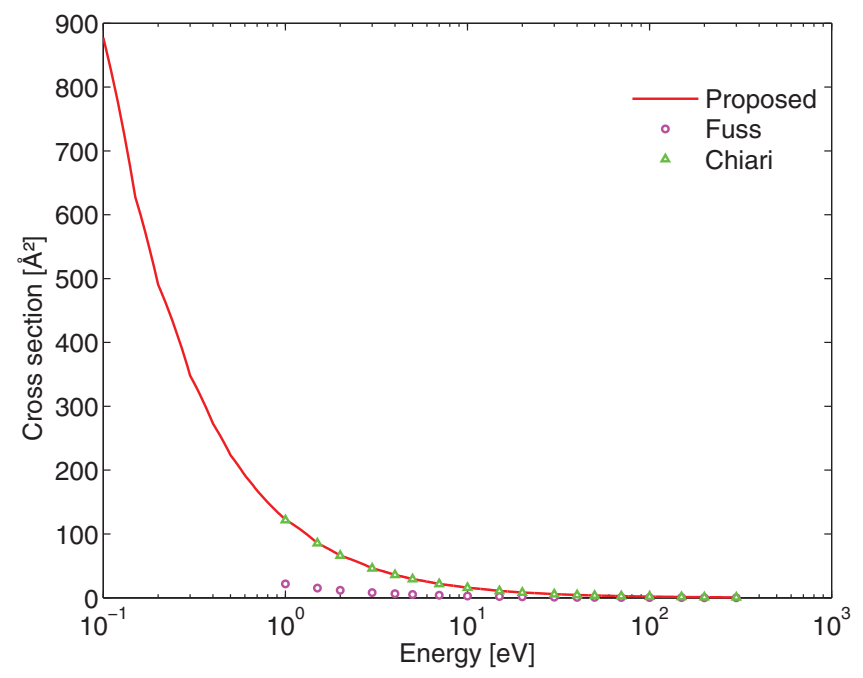

FIG. 9. (Color online) Proposed rotational ICS for electron scattering from THF, as compared with the estimation of Fuss et al. [25] and the calculated result of Chiari et al. [26].

This ICS is shown in Fig. 10 and we note that the uncertainty on its absolute values will be large given the process used to extract it. As can be seen from Fig. 10, we find a remnant ICS that has a relatively low-energy threshold and that is quite sharply peaked over a small energy domain. Such a behavior for the neutral dissociation process is found in some other targets [37-40], but it is very different to that observed in others (e.g., [41]) and what was previously extracted by Fuss et al. [25] for THF. In those latter studies the threshold energy for neutral dissociation is higher than what we find in Fig. 10, and the cross section extends over a wider energy region. As a consequence, we cannot be completely sure in correlating our remnant ICS to that for the neutral dissociation process; it might simply be an artifact of the process we used to extract it. Nonetheless, we do include our remnant ICS in our cross section database for calculation of the transport behavior of electrons in THF.

\section{TRANSPORT PROPERTIES OF ELECTRONS IN THF}

To model macroscopic systems, one needs complete and accurate cross-section sets. One of the key discriminative tests on the accuracy and completeness of cross-section sets is made through comparison of results from swarm experiments [27,28]. Electrons are released into a drift tube containing the gas and experience a spatially uniform electric field $(E)$. Completeness and accuracy of the cross-section set is investigated by correspondence of the measured transport coefficients with those calculated or simulated using that cross-section set. These transport coefficients include the drift velocity $W$, transverse and longitudinal diffusion coefficients $D_{T}$ and $D_{L}$, respectively, and the rate coefficients for a range of applied reduced fields $E / n_{0}$. It should be noted that $n_{0}$ is neutral gas density. In this section we implement the above set of proposed cross sections, to study the macroscopic transport properties of electron swarms in THF under typical swarm conditions $[27,28]$. We start with a brief description of which transport coefficients are measured and how one relates 


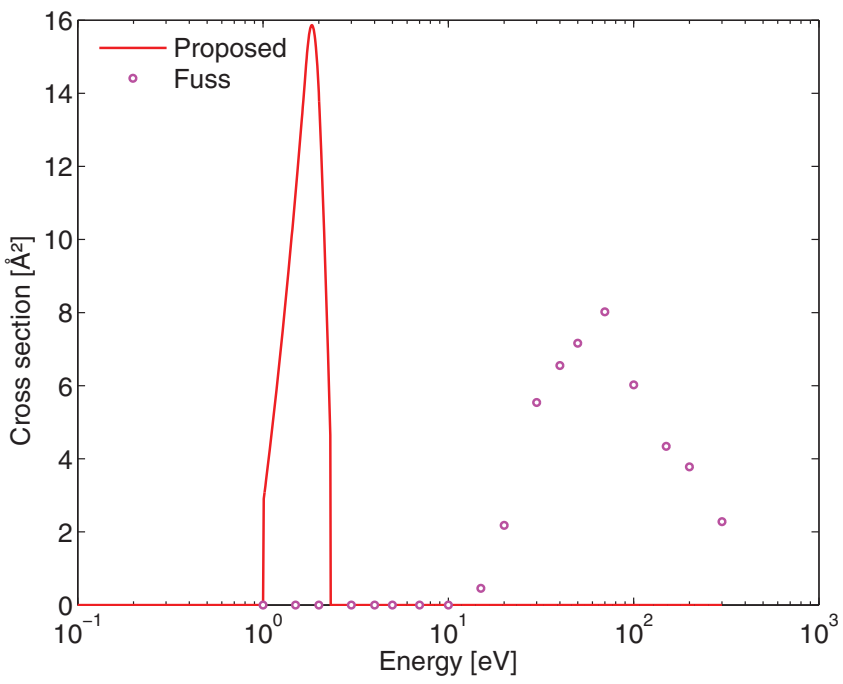

FIG. 10. (Color online) Proposed remnant ICS for THF, as compared with the earlier neutral dissociation ICS estimation of Fuss et al. [25].

them to the microscopic cross sections through an appropriate transport theory.

\section{A. Swarm transport coefficients}

Experimental swarm investigations of transport behavior are generally made by sampling charged particle currents or densities $n(\boldsymbol{r}, t)$. The connection between experiment and theory is generally made through the equation of continuity

$$
\frac{\partial n(\boldsymbol{r}, t)}{\partial t}+\nabla \cdot \boldsymbol{\Gamma}(\boldsymbol{r}, t)=S(\boldsymbol{r}, t),
$$

where $\boldsymbol{\Gamma}(\boldsymbol{r}, t)=n\langle\boldsymbol{c}\rangle$ is the electron flux and $S(\boldsymbol{r}, t)$ represents the production rate per unit volume per unit time arising from nonconservative collisional processes, such as ionization. In the hydrodynamic regime, the space-time dependence is projected onto functionals of the number density through a density gradient relation [42], and so the flux $\Gamma(\boldsymbol{r}, t)$ and source term $\boldsymbol{S}(\boldsymbol{r}, t)$ in Eq. (1) are expanded as

$$
\begin{gathered}
\boldsymbol{\Gamma}(\boldsymbol{r}, t)=\boldsymbol{W}_{F} n(\boldsymbol{r}, t)-\mathbf{D}_{F} \cdot \nabla n(\boldsymbol{r}, t)+\cdots, \\
\boldsymbol{S}(\boldsymbol{r}, t)=S^{(0)}-\boldsymbol{S}^{(1)} \odot \nabla n(\boldsymbol{r}, t)+\mathbf{S}^{(2)} \odot \nabla \nabla n(\boldsymbol{r}, t)+\cdots,
\end{gathered}
$$

where $\boldsymbol{W}_{F}$ is the $f l u x$ drift velocity and $\mathbf{D}_{F}$ is the flux diffusion tensor. These are often reported in the pulsed Townsend experiment configuration [43]. Substitution of expansions (2) and (3) into the continuity equation (1) yields the diffusion equation

$$
\frac{\partial n}{\partial t}+\boldsymbol{W} \cdot \nabla n-\mathbf{D}: \nabla \nabla n+\cdots=-R_{I} n,
$$

where $R_{i}=S^{(0)}$ is the loss rate and we define the bulk transport coefficients

$$
\begin{aligned}
\boldsymbol{W} & =\boldsymbol{W}_{F}+S^{(1)}, \\
\mathbf{D} & =\mathbf{D}_{F}+S^{(2)}
\end{aligned}
$$

Swarm experiments, such as the time-of-flight approach, are generally analyzed on the basis of the diffusion equation and hence the bulk coefficients, not the flux, are determined in those swarm experiments [44].

\section{B. Boltzmann equation and its solution}

The connection between the macroscopic transport properties discussed above and the microscopic processes governed by the cross-section set proposed is made through kinetic theory. Importantly, in swarm experiments, electric fields drive the electrons out of equilibrium with the background gas and hence the distribution of velocities of the electrons often becomes distinctly non-Maxwellian. In this case, the macro-micro connection must be made through a solution of Boltzmann's equation (or equivalently a Monte Carlo simulation). The motion of a dilute swarm of electrons (mass $\mathrm{m}$ ) moving through a background of dense neutral THF molecules in the presence of an applied electric field $E$ can be described by the linear Boltzmann equation

$$
\frac{\partial f}{\partial t}+c \cdot \nabla f+\frac{q \boldsymbol{E}}{m} \cdot \frac{\partial f}{\partial c}=-J\left(f, f_{0}\right),
$$

where $f(\boldsymbol{r}, \boldsymbol{c}, t)$ is the single-particle phase-space distribution function, which is a function of position $\boldsymbol{r}$, velocity $\boldsymbol{c}$, and time $t$. The acceleration on an electron of mass $m$ is due to the external homogeneous electric field $\boldsymbol{E}$. The collision operator $J\left(f, f_{0}\right)$ takes into account binary interactions between the electrons and the THF molecules, where $f_{0}$ denotes the THF molecule distribution function, which is assumed to be Maxwellian at the gas temperature $T_{0}$. The details of the collision operator used can be found in Ness et al. [45].

Equation (7) is an integro-differential equation for $f(\boldsymbol{r}, \boldsymbol{c}, t)$, a knowledge of which permits determination of all quantities of interest describing the behavior of the electron swarm. In the hydrodynamic regime in which swarm experiments are conducted, the space-time dependence of $f$ is assumed to have the form

$$
f(\boldsymbol{r}, \boldsymbol{c}, t)=\sum_{j=0} f^{(j)}(\boldsymbol{c}) \cdot(-\nabla)^{j} n(\boldsymbol{r}, t),
$$

where the $f^{(j)}$ are tensors of rank $j$, the dot denotes a $j$-fold scalar product and the local charged particle density at time $t$ is given by

$$
n(\boldsymbol{r}, t)=\int f(\boldsymbol{r}, \boldsymbol{c}, t) d \boldsymbol{c} .
$$

Substitution of the hydrodynamic expansion (8) into Eq. (7), and equating coefficients of the gradient expansion, results in a hierarchy of equations to solve for the velocity distribution functions $f^{(j)}(\boldsymbol{c})[46]$ :

$$
\frac{q \boldsymbol{E}}{m} \cdot \frac{\partial f^{(j)}}{\partial \boldsymbol{c}}+J\left(f^{(j)}, f_{0}\right)=\boldsymbol{c} f^{(j-1)}, \quad j=0,1, \ldots
$$

This is the microscopic picture. The solution of the hierarchy for the distribution functions $f^{(j)}(\boldsymbol{c})$ enables the calculation of the macroscopic measurable quantities through appropriate 
averages, e.g.,

$$
\begin{aligned}
\boldsymbol{W}_{F} & =\frac{1}{n} \int \boldsymbol{c} f^{(0)}(\boldsymbol{c}, t) d \boldsymbol{c}, \\
\mathbf{D}_{F} & =-\frac{1}{n} \int \boldsymbol{c} \boldsymbol{f}^{(1)}(\boldsymbol{c}, t) d \boldsymbol{c}, \\
\boldsymbol{S}^{(i)} & =\frac{1}{n} \int J_{R}\left(f^{(i)}, F_{0}\right) d \boldsymbol{c} .
\end{aligned}
$$

Solution of the hierarchy of kinetic equations (10) requires decomposition of $f^{(j)}(\boldsymbol{c})$ in velocity space. The first step in any analysis is typically the representation of the distribution function in terms of the directions of velocity space through an expansion in spherical harmonics [47],

$$
f^{(j)}(\boldsymbol{c}, t)=\sum_{l=0}^{\infty} \sum_{m=-l}^{l} f_{m}^{j(l)}(c, t) Y_{m}^{[l]}(\hat{\boldsymbol{c}}),
$$

where $Y_{m}^{[l]}(\hat{\boldsymbol{c}})$ are the spherical harmonics and $\hat{\boldsymbol{c}}$ denotes the angles of $\boldsymbol{c}$. While common practice is to set the upper bound of the $l$ summation to 1 (i.e., the two-term approximation) and consider only $m=0$ (i.e., a Legendre polynomial expansion), we do not make any such restrictive assumptions in this theory. In best practice, the integer $l_{\max }$ is successively incremented until a prescribed accuracy criterion is met, as considered below. This is a multiterm solution of Boltzmann's equation. Combining Eqs. (7) and (14) leads to the following hierarchy of coupled integro-differential equations for $f_{m}^{j(l)}$ :

$$
\begin{gathered}
\sum_{l^{\prime} m^{\prime}}\left\langle l m\left|\frac{e \boldsymbol{E}}{m} \cdot \frac{\partial}{\partial \boldsymbol{c}}+J\right| l^{\prime} m^{\prime}\right\rangle f_{m^{\prime}}^{j\left(l^{\prime}\right)} \\
=-\sum_{l^{\prime} m^{\prime}}\left\langle l m|\boldsymbol{c}| l^{\prime} m^{\prime}\right\rangle f_{m^{\prime}}^{j-1\left(l^{\prime}\right)} .
\end{gathered}
$$

Expressions for the matrix elements of the streaming operators are given in Robson et al. and Ness et al. [45,47]. The collision matrices, e.g., $\left\langle l m|J| l^{\prime} m^{\prime}\right\rangle=\left[J_{\text {elas }}^{l}+J_{\text {inel }}^{l}+\right.$ $\left.J_{\text {DEA }}^{l}+J_{\text {ion }}^{l}\right] \delta_{l^{\prime}, l} \delta_{m^{\prime}, m}$ (where the subscripts denote the relevant processes, and the inel encompasses rotational, vibrational, electronic excitations and neutral dissociation) are all diagonal in $l$ and $m$, since the collision operators are all scalars. Further representation of the speed dependence is required to solve the hierarchy of coupled operator equations. In this study we implement an expansion in terms of Sonine polynomials, and the reader is referred to White et al. [42] for further details on relating transport coefficients to the coefficients $f_{m}^{j(l)}$.

\section{Electron transport in THF}

In Figs. 11-13 we present results for swarm transport properties of electrons in THF, including the mean energy, drift velocity, diffusion, and rate coefficients. The results presented are in a quasi-steady state determined by a balance between power input from an applied electric field $E$ and energy-loss rate via collisions between electrons in the swarm and particles. All our results are presented as a function of the reduced electric field $E / n_{0}$, in the range $0.01-10000 \mathrm{Td}(1 \mathrm{Td}=1$ Townsend $=10^{-21} \mathrm{Vm}^{2}$ ). The temperature of the background gas of THF molecules is fixed at $293 \mathrm{~K}$.

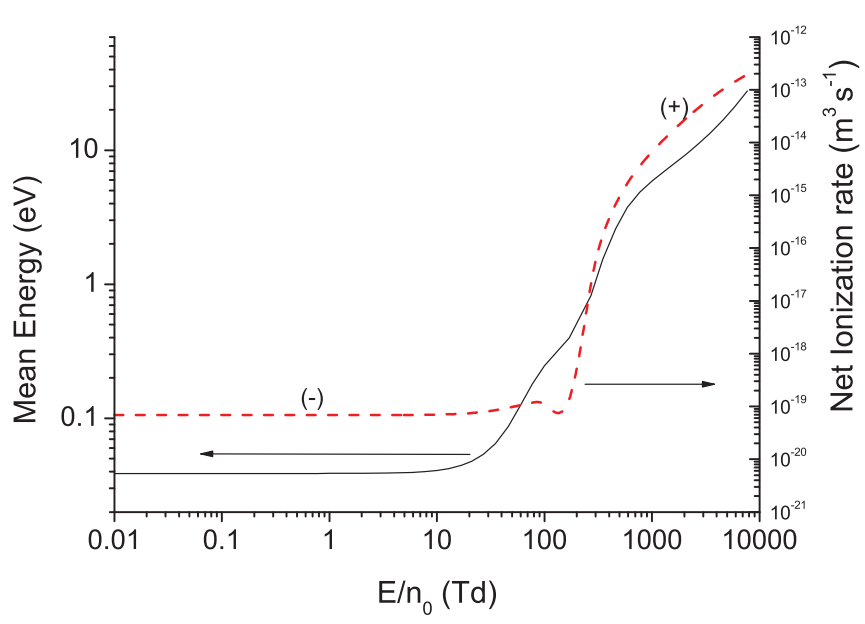

FIG. 11. (Color online) Mean energy and net ionization rate for electrons in THF as a function of the reduced electric field $(1 \mathrm{Td}=1$ Townsend $=10^{-21} \mathrm{Vm}^{2}$ ). Note the transition from a DEA-governed negative net ionization rate at lower $E / n_{0}$ [denoted by $\left.(-)\right]$ to an ionization-governed positive net ionization rate [denoted by $(+)]$ at higher $E / n_{0}$.

In the low field regime $\left(E / n_{0}<1 \mathrm{Td}\right)$, the electron swarm is essentially in thermal equilibrium with background THF molecules. As shown in Fig. 11 the mean energy is approximately equal to the background temperature and the field is a perturbation on the swarm's behavior. In this regime, the drift velocity (see Fig. 12) is essentially linear, representing an essentially constant mobility. Likewise, as displayed in Fig. 13 the diffusion is approximately equal to the thermal value and it is essentially isotropic $\left(D_{L} \approx D_{T}\right)$. DEA processes are operative in this field regime, as evidenced by the negative net ionization rate in Fig. 11. The magnitude is, however, small and so there are essentially no differences between the bulk and flux coefficients in this regime.

As we move to higher fields $\left(1 \mathrm{Td}<E / n_{0}<100 \mathrm{Td}\right)$, the thermal equilibrium state is finally broken. The rapidly falling cross-section magnitudes for elastic collisions results in a rapidly increasing mean energy with field in this regime,

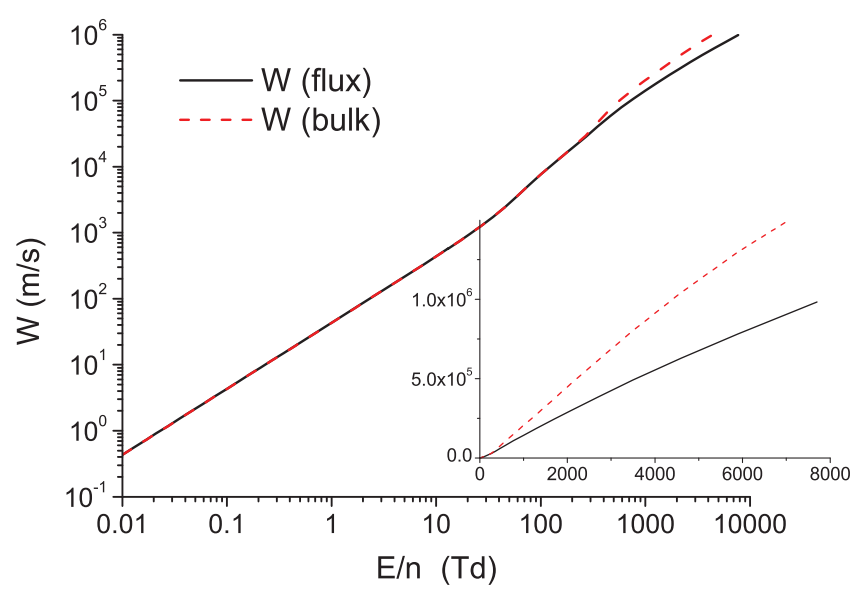

FIG. 12. (Color online) Bulk (dashed) and flux (solid) drift velocities for electrons in THF as a function of the reduced electric field. The inset uses a linear scale to emphasize the differences. 


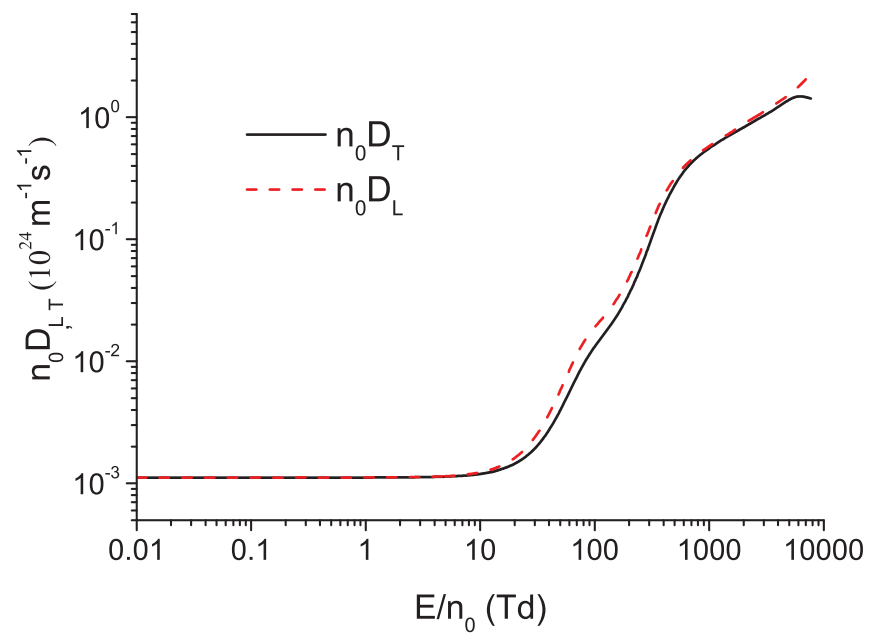

FIG. 13. (Color online) Reduced transverse and longitudinal flux diffusion coefficients for electrons in THF as a function of the reduced electric field.

as demonstrated in Fig. 11. This rapid rise in the mean energy is then slightly quenched by the neutral dissociation cross section and the ionization cross sections shown in Figs. 10 and 6 , which sees a plateauing of the mean energy above $\sim 100 \mathrm{Td}$ (see Fig. 11). The DEA rates rise slightly in this regime but the ionization eventually dominates the rates of the nonconservative processes in this regime. The behavior of the mean energy is also reflected in the diffusion coefficients presented in Fig. 13. While the diffusion coefficients have a thermal contribution to them, in this regime, we observe that diffusion becomes distinctly anisotropic (i.e., diffusion parallel and transverse to the electric field are distinctly different). As the thermal contribution to diffusion is relatively isotropic in this regime, this indicates that the primary source of anisotropic diffusion comes from the "differential velocity effect" [48], arising from a rapidly varying collision frequency with energy and spatial variation of the average energy through the swarm.

In the high field regime $\left(E / n_{0}>100 \mathrm{Td}\right)$, nonconservative effects associated with ionization (see Fig. 11) begin to impact on the transport properties. For the mean energy, the ionization process generates another electron with lower energy, resulting in a dilution of the energy and a reduced rate of increase with reduced field in the mean energy of the swarm. Importantly, there are explicit effects on the transport coefficients. In Fig. 12, we display both the flux and the bulk drift velocities. The flux represents the velocity averaged over all electrons in the swarm, while the bulk drift velocity represents the time rate of change of the center of mass of the electron swarm. Nonconservative processes have an implicit effect on the velocity distribution function and hence on the flux drift velocity. In particular, the nonconservative processes explicitly modify the center of mass of the swarm through nonuniform creation of electrons and consequently modify the bulk drift velocity. In Fig. 12, we observe that the bulk drift velocity is enhanced over the flux component, indicating that electrons are getting preferentially created at the front of the swarm and so shifting the center of mass in the direction of the field force.

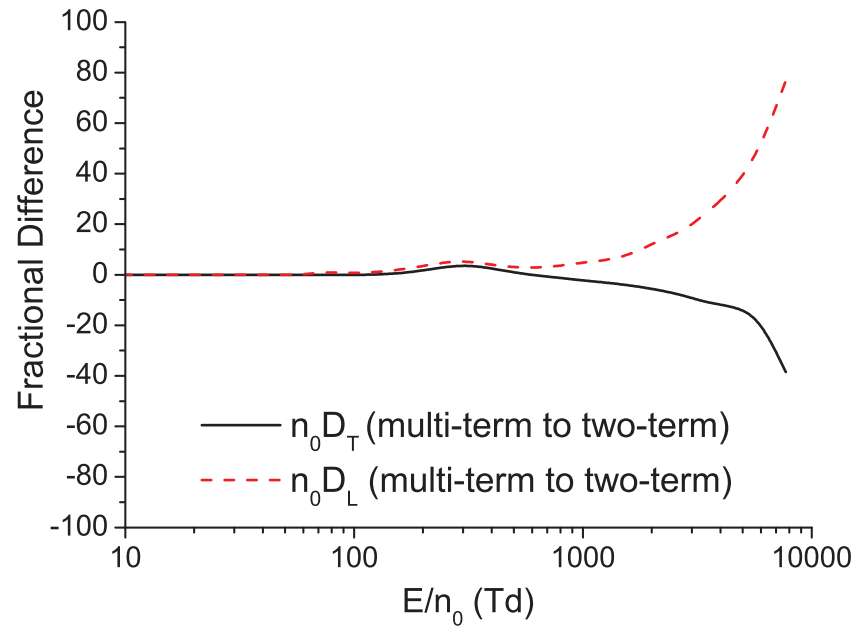

FIG. 14. (Color online) Percentage fractional difference of the multiterm to two-term approximations for the reduced flux transverse and longitudinal diffusion coefficients for electrons in THF as a function of the reduced electric field.

The two-term approximation has become commonplace in the calculation of electron transport properties in gases. In Fig. 14 we compare the two-term and multiterm approximations for the diffusion coefficients. For low fields, the two-term approximation is accurate to within $1 \%$ or better. As we move to higher fields, however, we observe that the two-term approximation can be in error by as much as $80 \%$. This is indicative of a highly anisotropic velocity distribution function. At high field, this anisotropy is evidenced by electron temperatures transverse and parallel to the electric field in THF differing by $30 \%$ or more. Analysis of swarm experiments at high fields will necessarily require a multiterm analysis or Monte Carlo simulation.

Finally, we make comment on the sensitivity of the transport coefficients to the details in the cross sections. The velocity distribution function is necessarily sensitive to changes in the cross-section set in certain field regimes, and the resulting transport coefficients, calculated via averages over the distribution function, can also be sensitive. The agreement in the cross sections between various experiments and with theory at high energies gives confidence in the calculated transport coefficients at higher fields. For low energies, however, where agreement between different experiments and experiments and theory are not satisfactory, or such cross-section data is nonexistent and requires extrapolation, importantly, swarm experiments may provide the only means to assess the accuracy and completeness of the cross-section set in this regime. The cross-section set can be further adjusted or tuned so that the calculated transport properties match the experimental swarm data. Measurements of the ionization rate will provide a further discriminative test of the ionization cross section (and implicitly on the other cross sections). Likewise, measurements of drift and diffusion coefficients will provide additional experimental tests on the MTCS and other cross sections. In the following section, we discuss the issues associated with electron swarm measurements in THF. 


\section{Swarm experiment considerations in THF using the pulsed-Townsend technique}

Several attempts were made to measure electron swarm coefficients (electron drift velocity, longitudinal diffusion coefficient and effective ionization coefficient) in THF using the pulsed Townsend technique [49,50]. Unfortunately, all of the attempts to measure electron avalanches in pure THF were unsuccessful because of a hitherto unobserved phenomenon.

THF vapor was injected into the evacuated discharge chamber to pressures close to $2 \times 10^{-6}$ Torr. We observed that the measured drift velocities did not reproduce for fixed $E / n_{0}$ and pressure. Large variations of up to $30 \%$ were observed in the measurement of the electron drift velocity $W$, which are far beyond our common uncertainties of $\pm 1 \%-2 \%$ in this coefficient. We followed these measurements with tests of drift velocities in pure $\mathrm{N}_{2}$, for which the electron swarm coefficients are well known. These results were in error. The vacuum vessel, electrode system, and all contacts and surfaces were all cleaned and washed to remove the layer of THF adhered to it. The system was retested with pure nitrogen, this time rendering values of $W$ which were within $\pm 1 \%-2 \%$ of the accepted values. Thus, it became clear that an effect due to the THF vapor in the discharge chamber was the cause of our failure. Our hypothesis is that a thin dielectric layer of THF forms on the cathode and anode surfaces, these becoming charged, thereby producing an additional electric field which affects the external one, and so changes the $E / n_{0}$ value across the discharge gap.

A second series of measurements in THF were performed after several months of successful measurements with other gases such as water vapor, $\mathrm{He}, \mathrm{N}_{2}$, and Ar. Initial measurements of $W$ in $1 \%$ THF in $\mathrm{N}_{2}$, over a range of applied reduced fields 5-45 Td, were found to be quite close to the pure $\mathrm{N}_{2}$ values. Subsequent measurements carried out $12 \mathrm{~h}$ later rendered $W$ values much higher than those measured previously. Drift velocity measurements in pure THF, in fact, produced such high values that they were difficult to understand.
A new discharge chamber has been built to measure electron swarm coefficients at higher $\left(25^{\circ} \mathrm{C}-120^{\circ} \mathrm{C}\right)$ and lower (down to $-20{ }^{\circ} \mathrm{C}$ ) temperatures. Our plan is to try and measure these coefficients at higher temperatures to avoid the formation of dielectric layers on the electrodes. Furthermore, we anticipate that measurements in low concentrations of THF mixtures with $\mathrm{N}_{2}$ or Ar, for instance, will be most feasible and avoid constant cleaning of the apparatus.

\section{CONCLUDING REMARKS}

In this paper, we have conducted a critical analysis of existing experimental and theoretical cross sections for electron scattering in $\mathrm{THF}$, to propose a largely complete set of cross sections in the energy range $\sim 0-300 \mathrm{eV}$. This has included the development of a set of "neutral dissociation" cross sections, for which no experimental measurements currently exist. This complete set of cross sections was then used to study the transport properties of electron swarms in THF, for a range of reduced electric fields from 0.01 to $10000 \mathrm{Td}$. The impact of the relevant cross sections on the transport properties has been discussed. We have also outlined issues associated with swarm measurements in THF, using current pulsed Townsend techniques, including a proposal for addressing them. We hope that this study serves to motivate further swarm experiments in order to provide a discriminating test on the accuracy and completeness of the THF cross-section set proposed.

\section{ACKNOWLEDGMENTS}

The authors would like to thank the Australian Research Council through its Centers of Excellence and Discovery programs for financial support, the Australian Academy of Science through its European Scientific Exchange Program, and the Spanish Ministerio de Economia y Productividad (Project FIS2012-32320). The experimental part was supported by Project No. PAPIIT IN 111611.
[1] B. Boudaïffa, P. Cloutier, D. Hunting, M. A. Huels, and L. Sanche, Science 287, 1658 (2000).

[2] B. Boudaïffa, D. Hunting, P. Cloutier, M. A. Huels, and L. Sanche, Int. J. Radiat. Biol. 76, 1209 (2000).

[3] Y. Itikawa and N. Mason, J. Phys. Chem. Ref. Data 34, 1 (2005).

[4] K. F. Ness, R. E. Robson, M. J. Brunger, and R. D. White, J. Chem. Phys. 136, 024318 (2012).

[5] R. D. White and R. E. Robson, Phys. Rev. Lett. 102, 230602 (2009).

[6] R. D. White, W. Tattersall, G. Boyle, R. E. Robson, S. Dujko, Z. Lj. Petrovic, A. Bankovic, M. J. Brunger, J. P. Sullivan, S. J. Buckman, and G. Garcia, Appl. Radiat. Isot. 83, 77 (2014).

[7] A. Zecca, C. Perazzolli, and M. J. Brunger, J. Phys. B 38, 2079 (2005).

[8] P. Możejko, E. Ptasinska-Denga, A. Domaracka, and C. Szmytkowski, Phys. Rev. A 74, 012708 (2006).
[9] C. J. Colyer, V. Vizcaino, J. P. Sullivan, M. J. Brunger, and S. J. Buckman, New J. Phys. 9, 41 (2007).

[10] M. Allan, J. Phys. B 40, 3531 (2007).

[11] M. G. P. Homem, R. T. Sugohara, I. P. Sanches, M. T. Lee, and I. Iga, Phys. Rev. A 80, 032705 (2009).

[12] W. Y. Baek, M. Bug, H. Rabus, E. Gargioni, and B. Grosswendt, Phys. Rev. A 86, 032702 (2012).

[13] A. Gauf, L. R. Hargreaves, A. Jo, J. Tanner, M. A. Khakoo, T. Walls, C. Winstead, and V. McKoy, Phys. Rev. A 85, 052717 (2012)

[14] M. Dampc, A. R. Milosavljević, I. Linert, B. P. Marinković, and M. Zubek, Phys. Rev. A 75, 042710 (2007).

[15] M. Dampc, I. Linert, A. Milosavljević, and M. Zubek, Chem. Phys. Lett. 443, 17 (2007)

[16] M. A. Khakoo, D. Orton, L. R. Hargreaves, and N. Meyer, Phys. Rev. A 88, 012705 (2013).

[17] T. P. T. Do, M. Leung, M. Fuss, G. Garcia, F. Blanco, K. Ratnavelu, and M. J. Brunger, J. Chem. Phys. 134, 144302 (2011). 
[18] M. Zubek, M. Dampc, I. Linert, and T. Neumann, J. Chem. Phys. 135, 134317 (2011)

[19] M. Fuss, A. Muñoz, J. C. Oller, F. Blanco, D. Almeida, P. LimãoVieira, T. P. D. Do, M. J. Brunger, and G. García, Phys. Rev. A 80, 052709 (2009).

[20] M. Dampc, E. Szymanska, B. Mielewska, and M. Zubek, J. Phys. B 44, 055206 (2011).

[21] D. Bouchiha, J. D. Gorfinkiel, L. G. Caron, and L. Sanche, J. Phys. B 39, 975 (2006).

[22] C. Winstead and V. McKoy, J. Chem. Phys. 125, 074302 (2006).

[23] C. S. Trevisan, A. E. Orel, and T. N. Rescigno, J. Phys. B 39, L255 (2006).

[24] P. Mozejko and L. Sanche, Radiat. Phys. Chem. 73, 77 (2005).

[25] M. C. Fuss, R. Colmenares, A. G. Sanz, A. Muñoz, J. C. Oller, F. Blanco, T. P. T. Do, M. J. Brunger, D. Almeida, P. LimãoVieira et al., J. Phys.: Conf. Ser. 373, 012010 (2012).

[26] L. Chiari, E. Anderson, W. Tattersall, J. R. Machacek, P. Palihawadana, C. Makochekanwa, J. P. Sullivan, G. García, F. Blanco, R. P. McEachran et al., J. Chem. Phys. 138, 074301 (2013).

[27] L. G. H. Huxley and R. W. Crompton, The Drift and Diffusion of Electrons in Gases (Wiley, New York, 1974).

[28] Z. L. Petrovic, S. Dujko, D. Maric, G. Malovic, Z. Nikitovic, O. Sasic, J. Jovanovic, V. Stojanovic, and M. RadmilovicRadenovic, J. Phys. D 42, 194002 (2009).

[29] R. E. Robson, R. D. White, and K. F. Ness, J. Chem. Phys. 134, 064319 (2011).

[30] A. G. Sanz, M. C. Fuss, F. Blanco, J. D. Gorfinkiel, D. Almeida, F. F. da Silva, P. Limao-Vieira, M. J. Brunger, and G. Garcia, J. Chem. Phys. 139, 184310 (2013).

[31] M. C. Fuss, A. G. Sanz, F. Blanco, J. C. Oller, P. Limao-Vieira, M. J. Brunger, and G. Garcia, Phys. Rev. A 88, 042702 (2013).

[32] M. A. Khakoo, H. Silva, J. Muse, M. C. A. Lopes, C. Winstead, and V. McKoy, Phys. Rev. A 87, 049902(E) (2013).
[33] A. Faure, J. D. Gorfinkiel, and J. Tennyson, Mon. Not. R. Astron. Soc. 347, 323 (2004).

[34] K. Aflatooni, A. M. Scheer, and P. D. Burrow, J. Chem. Phys. 125, 054301 (2006).

[35] A. Jain, J. Phys. B 21, 905 (1988).

[36] US National Library of Medicine (2013), http://chem.sis. nlm.nih.gov/chemidplus.

[37] K. Anzai, H. Kato, M. Hoshino, H. Tanaka, Y. Itikawa, L. Campbell, M. J. Brunger, S. J. Buckman, H. Cho, F. Blanco, G. Garcia, P. Limão-Vieira, and O. Ingólfssson, Eur. Phys. J. D 66, 1 (2012).

[38] Y. Itikawa, J. Phys. Chem. Ref. Data 31, 749 (2002).

[39] Y. Itikawa, J. Phys. Chem. Ref. Data 38, 1 (2009).

[40] A. Zecca, G. P. Karwasz, and R. S. Brusa, Phys. Rev. A 45, 2777 (1992).

[41] P. C. Cosby, J. Chem. Phys. 98, 9544 (1993).

[42] R. D. White, R. E. Robson, S. Dujko, P. Nicoletopoulos, and B. Li, J. Phys. D 42, 194001 (2009).

[43] G. Ruíz-Vargas, M. Yousfi, and J. de Urquijo, J. Phys. D 43, 455201 (2010).

[44] R. E. Robson, R. D. White, and Z. L. Petrović, Rev. Mod. Phys. 77, 1303 (2005).

[45] K. F. Ness and R. E. Robson, Phys. Rev. A 34, 2185 (1986).

[46] K. Kumar, Aust. J. Phys. 33, 449 (1980).

[47] R. E. Robson and K. F. Ness, Phys. Rev. A 33, 2068 (1986).

[48] R. D. White, K. F. Ness, R. E. Robson, and B. Li, Phys. Rev. E 60, 2231 (1999).

[49] O. Šašić, J. Jovanović, Z. L. Petrović, J. de Urquijo, J. Castrejón, J. L. Hernández-Ávila, and E. Basurto, Phys. Rev. E 71, 046408 (2005).

[50] S. Dupljanin, J. de Urquijo, O. Šašić, E. Basurto, A. M. Juárez, J. L. Hernández-Ávila, S. Dujko, and Z. L. Petrović, Plasma Sources Sci. Technol. 19, 025005 (2010). 